\title{
Magnetic dissipation force microscopy
}

\author{
P. Grütter, ${ }^{\text {a) }}$ Y. Liu, and P. LeBlanc \\ Department of Physics, Centre for the Physics of Materials, McGill University, Montréal H3A 2T8, Canada \\ U. Dürig \\ Zurich Research Laboratory, IBM Research Division, CH-8803 Rüschlikon, Switzerland
}

(Received 4 December 1996; accepted for publication 6 May 1997)

\begin{abstract}
A method of measuring magnetic dissipation on a sub-100 nm scale is presented. This technique relies on measuring changes in the damping of the oscillating tip in a magnetic force microscope (MFM). Damping contrast is strongly correlated with micromagnetic structure and in the case of $\mathrm{NiFe}$, is in quantitative agreement with magnetoelastic losses in the sample. On recording tracks, large damping signals are observed. This has direct consequences on the interpretation of traditional MFM images acquired with detectors that convolute frequency and damping information. (C) 1997 American Institute of Physics. [S0003-6951(97)01428-9]
\end{abstract}

In magnetic force microscopy (MFM), the interaction of a magnetic tip with the stray field of a sample is measured. ${ }^{1}$ For a known tip magnetization direction, the measured interaction can be interpreted in terms of the sample magnetic domain structure. ${ }^{2}$ An implicit assumption often made is to neglect any distortions of the domain structure as a result of the tip stray field. In this letter, we present a method of directly quantifying the influence of the tip stray field on the sample domain structure. We do this by measuring the damping of the oscillating tip in a MFM simultaneously with the usual frequency shifts associated with tip-sample force gradient variations. A change in damping of the MFM probe is the result of energy transfered between the tip and the sample and is detected as a difference in cantilever oscillation amplitude. Damping due to Joule dissipation in semiconductors has previously been measured with a different detection scheme by Denk and Pohl. ${ }^{3}$

For these experiments, we use a home-built MFM with a fiber-optic interferometric deflection sensor that can be operated in moderate vacuum $\left(10^{-6} \mathrm{mbar}\right)$. We use commercially available microfabricated $\mathrm{Si}$ or $\mathrm{Si}_{3} \mathrm{~N}_{4}$ force sensors with typical resonance frequencies of $\omega / 2 \pi=30 \mathrm{kHz}$, spring constants of $k=0.1 \mathrm{~N} / \mathrm{m}$, a mechanical $Q$ of 30 in air and $600-8000$ in vacuum. They are sputter coated with $15-100$ $\mathrm{nm} \mathrm{CoPtCr}$ and magnetized in a $1 \mathrm{~T}$ field along their tip axis prior to being used in the MFM. ${ }^{4}$ All data discussed here were acquired in the constant force gradient mode ${ }^{5}$ using the phase controlled oscillator method ${ }^{6}$ with cantilever oscillation amplitudes $A=10 \mathrm{~nm}$. Our detection electronics centers around a home-built phased locked loop (PLL) circuit. ${ }^{7}$ With the PLL we track the force sensor resonant frequency with an accuracy of $0.1 \mathrm{~Hz}$ in a $1 \mathrm{kHz}$ bandwidth. For the force sensors used, the detection sensitivity is dominated by thermal noise of the force sensor (which is about ten times larger than the $10^{-6} \mathrm{~N} / \mathrm{m}$ limit due to electronic noise on the PLL output).

When the PLL is locked, associated electronics produce an output drive signal $A^{\prime}$ at exactly the measured resonant frequency of the cantilever with a well defined, adjustable phase. The amplitude of this drive signal is controlled by a separate feedback circuit and is acquired simultaneously with

${ }^{a)}$ Electronic mail: grutter@physics.mcgill.ca the force gradient feedback signal. The cantilever oscillation amplitude $A$ is a direct measure of the damping $\gamma$ or the mechanical $Q$ factor $(\gamma=k / \omega Q)$. Since $A=\left(F_{0} / \omega \cdot Q\right)$, where $F_{0} \sim A^{\prime}$ is the driving force, we observe changes $\delta Q$ as a result of changes in the oscillation amplitude, i.e., $\delta Q=Q \cdot \delta A^{\prime} / A^{\prime}$. The drive feedback circuit adjusts $A^{\prime}$ to maintain a constant oscillation amplitude $A$. Measurements of $A$ are thermally limited and thus also affect $Q$ measurements. The thermal noise $\delta Q_{\text {thermal }}$ is given by $\delta Q_{\text {thermal }}=(1 / A) \sqrt{2 k_{B} T \cdot Q^{3} \cdot B W / k \cdot \omega}$ with $k_{B}$ the Boltzmann factor, $T$ the temperature, and $B W$ the measurement bandwidth. For our cantilevers $\delta Q_{\text {thermal }}=0.02$ in air and $\delta Q_{\text {thermal }}=1.8$ in vacuum with $B W=35 \mathrm{~Hz}$. All dissipation data presented here are thermally limited. A better signal to noise is predicted and indeed observed by measuring dissipation in vacuum.

Crucial to a meaningful damping measurement is a minimal phase error and minimal cross-talk between the different feedback loops involved. Frequency dependent phase shifts (e.g., due to the detection electronics or filters) will drive the cantilever off resonance. A larger drive amplitude $A^{\prime}$ would then be necessary to maintain $A=$ const, which would be falsely interpreted as a change in cantilever damping. Our carefully optimized electronics maintain a phase shifts of less than 0.003 for input frequencies between $10 \mathrm{kHz}$ and 2 MHz. This amounts to an error of $\delta Q<0.0001$, substantially smaller than the thermal limit. A second source of phase shifts are frequency errors, e.g., as a result of varying force gradients between tip and sample (i.e., the MFM feedback is not tracing lines of perfectly constant force gradients). The drive output consequently excites the cantilever offresonance, again mimicking a change in damping. The influence of frequency errors on the drive output can be measured in situ by modulating the PLL reference frequency. This also allows us to verify that the two feedback loops of the PLL are indeed uncoupled by demodulating a FM (or AM) reference signal while monitoring the frequency and $Q$ outputs. A FM input signal of constant amplitude should not induce any change on the $Q$ output of the detection electronics. A nonobservable (i.e., noise limited) cross-talk is necessary for a meaningful interpretation of measurements. We determined $\delta Q<0.004$ for a $10 \mathrm{~Hz}$ modulation (substantially larger than the typical frequency feedback errors of $1-2 \mathrm{~Hz}$ ). A further 

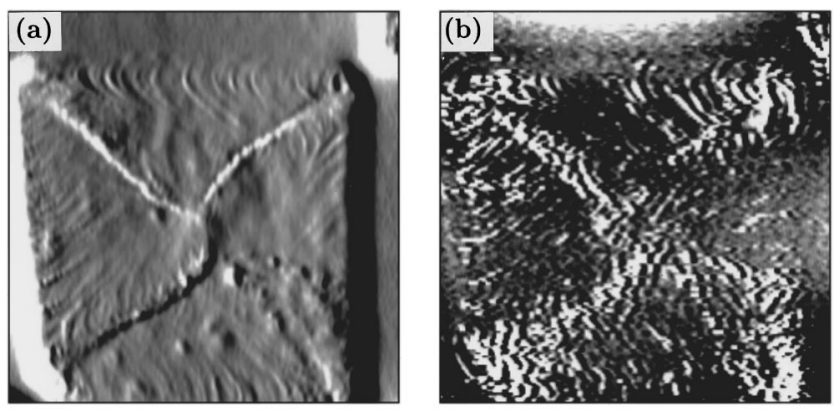

FIG. 1. (a) Constant force gradient image of a $30 \mathrm{~nm}$ thick, $20 \mu \mathrm{m} \mathrm{NiFe}$ square imaged at $p=10^{-6}$ mbar. To enhance details, the image was differentiated along the fast scan direction. (b) Dissipation image acquired simultaneously with (a). White corresponds to a larger cantilever damping, full scale variation in this image corresponds to $9 \mathrm{pNs} / \mathrm{m}(\Delta Q / Q=150 / 8000)$. No image processing was applied to this data.

potential phase error is particular to our fiber optic interferometric deflection sensing technique. de deflections $\Delta z$ as a result of forces acting on the cantilever will result in an optical path length difference and thus a phase shift $\Delta \phi=(2 \Delta z / \lambda) \cdot 2 \pi$. In our experiments we monitored this deflection simultaneously with the damping, the force gradient signal and the variations in force gradient (=MFM feedback frequency error). The maximum total deflection was always smaller than $1 \mathrm{~nm}$ and thus introduced a phase error of 0.01 . In conclusion, by carefully designing and characterizing our PLL, keeping the MFM feedback errors to $2 \mathrm{~Hz}$ and controlling phase errors due to optical path length changes, we are confident that all measured drive amplitude changes are indeed due to a change in cantilever damping. It is crucial to quantify frequency dependent phase shifts and cross-talk between frequency and amplitude channels in any detection system employed in damping measurements. Two channel digital lock-in amplifiers have similar characteristics as the described PLL, although it is problematic to use them with high- $Q$ cantilevers. ${ }^{8}$

The MFM feedback loop adjusts $z$ in order to maintain a constant force gradient. As cantilever damping is dominated by viscous drag of air at atmospheric pressures, a change in $z$ can lead to variations in damping of only indirect magnetic origin. By operating the MFM in vacuum, viscous drag of air becomes negligible and the $Q$ factor changes only when $z<20 \mathrm{~nm}$. Vacuum operation of the MFM also greatly increases sensitivity.

As a first test sample, we selected a $30 \mathrm{~nm}$ thick NiFe sample sputtered onto Si with a coercivity of 2 Oe and patterned into $20 \mu \mathrm{m}$ squares. Figure 1(a) shows a constant force gradient image $\left(F^{\prime}=1 \cdot 10^{-4} \mathrm{~N} / \mathrm{m}\right)$ of such an NiFe square acquired at an average tip-sample separation of $70 \mathrm{~nm}$ in a vacuum of $10^{-6}$ mbar. These images were acquired in about 10 min with a $20 \mathrm{~nm}$ thin $\mathrm{CoPtCr}$ coated tip. The image of the NiFe square is distorted due to uncorrected piezo nonlinearities. The expected well-defined domain structure is clearly observed. By simultaneously acquiring the reverse scan signal, we verified that under these imaging conditions no influence of the tip stray field on the domain structure is detectable in the constant force gradient image [Fig. 1(a)]. The simultaneously acquired damping signal [Fig. 1(b)] shows pronounced maxima correlated with the domain wall positions. The increased sensitivity and resolution associated with vacuum operation of the MFM allows the observation of a magnetic ripple structure localized at the edge of the NiFe square in Figs. 1(a) and 1(b). Signs of a similar ripple structure on $\mathrm{NiFe}$ squares have previously been observed by Mamin et al. ${ }^{9}$ It is interesting to notice that damping contrast is maximized at the square edges (in particular the corners), whereas contrast is below the noise level at the intersection of the domain walls at the center of the square. The $Q$ factor changed by $150(\Delta Q / Q=150 / 8000)$ when the tip is scanned across a wall. This corresponds to a power dissipation of $6 \cdot 10^{-18} \mathrm{~W}$. The width of these maxima shows a strong dependence on tip-sample separation $z$, becoming wider and less pronounced at larger separations.

An immediate question is which mechanisms are responsible for the observed magnetic damping, which can be seen as the equivalent to the area (=loss) of a local minor hysteresis loop. Damping due to tip-induced flux changes at the sample will only lead to localized damping contrast if the induced eddy current encounters a spatially inhomogeneous resistance. Eddy current damping of wall oscillations, which are induced by the vibrating magnetic tip, are $10^{6}-10^{7}$ times smaller than the observed effect. ${ }^{10}$ Eddy current damping of the tip oscillations as a result of the stronger local sample stray field at domain walls are also of the same small magnitude. ${ }^{10}$ The tip induced rotation of sample spins and subsequent dragging along of a domain wall as suggested by micromagnetic simulations ${ }^{11}$ and a model neglecting exchange interactions ${ }^{12}$ need to be investigated quantitatively. Macroscopically, this contrast mechanism is equivalent to a measurement of the out-of-phase component of the magnetic susceptibility. It is expected that the ease of rotation of spins (which is coupled to the micromagnetic environment) leads to a cantilever damping that shows lateral variations that are both larger and smaller than the average damping associated with this mechanism. A related contrast mechanism-lateral variations of the in-phase component of the magnetic susceptibility-has recently been reported. ${ }^{13}$

Coherent generation of phonons via magnetostriction reproduces some of the experimental data both qualitatively and quantitatively. ${ }^{10}$ Generation of phonons is an inevitable consequence of the tip stray field influencing the sample micromagnetic structure. Recall that the domain wall thickness depends on the exchange energy, the anisotropy energy and the magnetostatic energy. The latter is a function of the external field, which in this case is provided by the tip and thus has both a dc and an ac component. The ac component leads to oscillations (of typically a few nanometers) of the wall width at $\omega$. When the thickness of the wall changes, strain due to magnetoelastic coupling will occur. The vibrating domain wall thus dissipates energy by emitting sound waves at the cantilever resonant frequency. In this model, the energy dissipated in one cycle is of the order of $10^{-21} \mathrm{~J}$ per oscillation cycle and in excellent agreement with the experimental results on $\mathrm{NiFe}$.

Dissipation is quadratic in the magnetostriction coefficient. Indeed, on thin films of Terfenol-D, ${ }^{14}$ with a magnetostriction about 100 times larger than $\mathrm{NiFe}$, we measured a corresponding increase in damping. Furthermore, we observed a minimum of the damping at the center of 

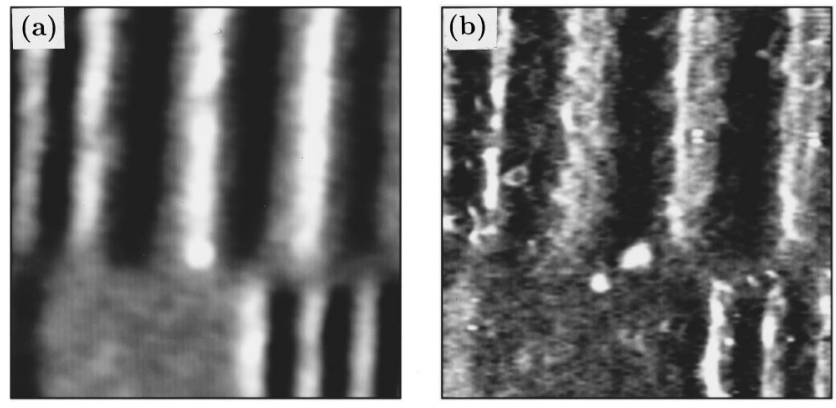

FIG. 2. (a) Constant force gradient image of tracks written in a $\mathrm{CoPtCr}$ longitudinal magnetic recording medium. Image size is $10 \mu \mathrm{m}$ by $10 \mu \mathrm{m}$, maximum $z$ variations are $15 \mathrm{~nm}$. No image processing except for a plane subtraction was performed. (b) Raw data of the simultaneously acquired cantilever dissipation. Full scale variation corresponds to $45 \mathrm{pNs} / \mathrm{m}$ $(\Delta Q / Q=30 / 8000)$.

Terfenol-D walls. This can be qualitatively understood as Terfenol-D has a perpendicular anisotropy with Néel-type walls (which couple strongly to $H_{x}^{\text {tip }}$, which has a minima at the tip center). This is in contrast to $\mathrm{NiFe}$ walls, where maxima in dissipation are observed due to the coupling to $H_{z}^{t i p}$. A tenfold increase in damping is expected for magnetic recording media (magnetostriction coefficients about three times larger than $\mathrm{NiFe}$ ). To verify this, we imaged tracks written in a $40 \mathrm{~nm}$ thick $\mathrm{CoPtCr}$ recording medium with identically the same tip as used in Figs. 1(a) and 1(b). Indeed, a very strong damping signal (five times larger) was observable that showed good correlation with the simultaneously measured magnetic structure [Figs. 2(a) and 2(b), respectively]. It is worthwhile to notice that the damping image actually shows more details and even seems to have a higher resolution than the force gradient image. Averaged line scans along a track however clearly show a qualitative difference (also observed with several other tips) when compared to NiFe: dissipation profiles show not only maxima, but also minima correlated with the magnetization transition regions.

Phenomenologically, this contrast is a result of the demagnetization field associated with the recorded bits. The demagnetization field sign depends on whether the bit is a North or a South pole. The hysteresis loop for the magnetic material is shifted by this demagnetization field. The MFM tip also produces a field of a given sign, adding to the demagnetization field for one sign bit and subtracting for the other. Since the damping contrast essentially is due to the MFM tip moving the sample magnetization around a minor hysteresis loop, the gray background in Fig. 2(b) is associated with the minor hysteresis loop of an unshifted loop. The losses in the transition regions can either be greater or smaller than this average as a result of the demagnetization field associated with the bit.

Microscopically, we presently do not quantitatively understand the origin of the dissipation in these minor hyster- esis loops of CoPtCr. The observed damping contrast is not due to eddy current damping nor the sample stray field influencing the tip magnetic structure. The former was discarded by attempting to image with a $100 \mathrm{~nm} \mathrm{Cu}$ coated force sensor. No damping signal was observed in accordance with simulations. ${ }^{10}$ The latter was verified by using different tips as well as by imaging the domain structure and measuring the damping signal on a $4 \mathrm{~nm}$ thin Co film with very small stray field variations, where a similar contrast was observed. Irrespective of the origin, an immediate consequence of the large damping signal observed on the magnetic recording tracks is that any detector with a frequency dependent phase response (such as most lock-in based systems) will convolute force gradient with damping information. This makes quantitative MFM data interpretation difficult.

In conclusion, we present a new operation mode of MFM-magnetic dissipation imaging. Simultaneously to constant force gradient MFM data we measure changes in force sensor damping as a result of energy transferred between the tip and sample. The magnitude of the damping signal depends very sensitively on the micromagnetic sample structure, thus making it a potentially powerful tool in micromagnetic investigations.

The authors acknowledge useful discussions with B. Ellman and initial help with the electronics by F. Battiston and H.-R. Steinauer. The sample of Terfenol-D was kindly provided by Q. Su and M. Wuttig, Dept. of Materials and Nuclear Engineering, University of Maryland. This work was supported by grants from the National Science and Engineering Research Council of Canada and Le Fonds pour la Formation des Chercheurs et l'Aide à la Recherche de la Province de Québec.

${ }^{1}$ For reviews see: P. Grutter, H.J. Mamin, and D. Rugar, Springer Series Surf. Sci. 28, 151 (1992); K. Babcock, M. Dugas, S. Manalis, and V. Elings, Mater. Res. Soc. Symp. Proc. 355, 311 (1995).

${ }^{2}$ P. Grutter and R. Allenspach, Geophys. J. Int. 116, 502 (1994).

${ }^{3}$ W. Denk and D.W. Pohl, Appl. Phys. Lett. 59, 2171 (1991).

${ }^{4}$ P. Grutter, D. Rugar, H.J. Mamin, G. Castillo, S.E. Lambert, C.-J. Lin, R.M. Valetta, O. Wolter, T. Bayer, and J. Greschner, Appl. Phys. Lett. 57, 1820 (1990).

${ }^{5}$ Because of the rather large oscillation amplitude the term force gradient refers to an averaged force gradient that is sampled by the tip during one oscillation cycle.

${ }^{6}$ U. Durig, O. Zuger, and A. Stalder, J. Appl. Phys. 72, 1778 (1992).

${ }^{7}$ U. Durig and H.R. Steinauer (unpublished).

${ }^{8}$ T.R. Albrecht, P. Grutter, D. Horne, and D. Rugar, J. Appl. Phys. 69, 668 (1991).

${ }^{9}$ H.J. Mamin, D. Rugar, J.E. Stern, R.E. Fontana, Jr., and P. Kasiraj, Appl. Phys. Lett. 55, 318 (1989).

${ }^{10}$ Y. Liu, B. Ellmann, and P. Grutter, Appl. Phys. Lett. (submitted).

${ }^{11}$ M.R. Scheinfein, J. Unguris, D.T. Pierce, and R.J. Celotta, J. Appl. Phys. 67, 5932 (1990).

${ }^{12}$ D.W. Abraham and F.A. McDonald, Appl. Phys. Lett. 56, 1181 (1990).

${ }^{13}$ S. Foss, R. Proksch, E.D. Dahlberg, B. Moskowitz, and B. Walsh, Appl. Phys. Lett. 69, 3426 (1996).

${ }^{14}$ Q. Su, Y. Zheng, A. Roytburd, and M. Wuttig, Appl. Phys. Lett. 66, 2424 (1995) 